\title{
Wide-gap Semiconducting Graphene from Nitrogen-seeded SiC
}

\author{
F. Wang, ${ }^{1}$ G. Liu, ${ }^{2}$ S. Rothwell, ${ }^{3}$ M. Nevius, ${ }^{4,5}$ A. Tejeda, ${ }^{4,5}$ A. Taleb-Ibrahimi, ${ }^{6}$ L.C. Feldman, ${ }^{6}$ P.I. Cohen, ${ }^{6}$ and E.H. Conrad ${ }^{6}$ \\ ${ }^{1}$ School of Physics, The Georgia Institute of Technology, Atlanta, Georgia 30332-0430, USA \\ ${ }^{2}$ Institute for Advanced Materials Devices and Nanotechnology, Rutgers University, Piscataway, New Jersey 08854 \\ ${ }^{3}$ Department of Electrical and Computer Engineering, \\ University of Minnesota, Minneapolis, Minnesota 55455 \\ ${ }^{4}$ Institut Jean Lamour, CNRS - Univ. de Nancy - UPV-Metz, 54506 Vandoeuvre les Nancy, France \\ ${ }^{5}$ Synchrotron SOLEIL, L'Orme des Merisiers, Saint-Aubin, 91192 Gif sur Yvette, France \\ ${ }^{6}$ UR1 CNRS/Synchrotron SOLEIL, Saint-Aubin, 91192 Gif sur Yvette, France
}

\begin{abstract}
All carbon electronics based on graphene has been an elusive goal. For more than a decade, the inability to produce significant band-gaps in this material has prevented the development of semiconducting graphene. While chemical functionalization was thought to be a route to semiconducting graphene, disorder in the chemical adsorbates, leading to low mobilities, have proved to be a hurdle in its production. We demonstrate a new approach to produce semiconducting graphene that uses a small concentration of covalently bonded surface nitrogen, not as a means to functionalize graphene, but instead as a way to constrain and bend graphene. We demonstrate that a submonolayer concentration of nitrogen on $\mathrm{SiC}$ is sufficient to pin epitaxial graphene to the $\mathrm{SiC}$ interface as it grows, causing the graphene to buckle. The resulting 3-dimensional modulation of the graphene opens a band-gap greater than $0.7 \mathrm{eV}$ in the otherwise continuous metallic graphene sheet.
\end{abstract}

PACS numbers: 73.22.Pr, 61.48.Gh, 79.60.-i

Keywords: Graphene, Graphite, SiC, Silicon carbide, Graphite thin film, dopants

The goal of developing all-carbon electronics requires the ability to charge dope graphene and convert it between metallic and wide band-gap semiconducting forms. While doping graphene by adsorbates or more elaborate chemical means has made rapid progress, $1 \cdot 7$ opening a band-gap in graphene has been problematic. Two routes to wide-band-gap semiconducting graphene have been pioneered: electron confinement and chemical functionalization. Electron confinement in lithographically patterned narrow graphene ribbons has been plagued by lithographic limits and edge disorder, $\stackrel{8-11}{r}$ although recent results from sidewall grown graphene ribbon are showing new progress that could lead to band-gaps larger than $0.6 \mathrm{eV} !^{[12] 13}$ Functionalized graphene band-gaps can be produced by imposing a periodic potential in the graphene lattice through ordered chemical adsorption $14 \mid 15$ or ordered substitutional impurities replacing carbon atoms. 16

In this work we demonstrate a novel approach to band-gap engineering in graphene using a nitrogen seeded $\mathrm{SiC}$ surface. Rather than using chemical vapor deposition (CVD) in an ammonia atmosphere or nitrogen plasmas to dope graphene by post seeding the films with nitrogen, $5[7 \mid 17$ we show that a submonolayer concentration of nitrogen adsorbed on $\mathrm{SiC}$ prior to graphene growth causes a large band-gap to open in the subsequently grown, continuous graphene sheets. Using X-ray photoemission spectroscopy (XPS), scanning tunneling microscopy (STM), and angle resolved photoemission spectroscopy (ARPES), we show that a submonolayer concentration of bonded nitrogen at the graphene-SiC interface leads to a $0.7 \mathrm{eV}$ semiconducting form of graphene.

The band-gap is not due to chemical functionalization since the small concentrations used in these studies are expected to have little effect on graphene's electronic band structure.16]18
Instead, STM topographs and $d I / d V$ images, showing that the graphene is buckled into folds with $1-2 \mathrm{~nm}$ radii of curvature, suggests two possible origins for the gap: either a quasiperiodic strain $\frac{19}{19}$ or electron localization in the $1-2 \mathrm{~nm}$ wide buckled ribbons ${ }^{20}$ We note that buckled graphene is synonymously described as "folds", "wrinkles", and "pleats" in the literature. We use these descriptions interchangeably in this paper.

To produce this semiconducting form of graphene, we predeposit nitrogen onto a $\mathrm{SiC}(000 \overline{1})$ surface [see experimental details and supplemental material]. After nitrogenation, there is a strong N1s peak at 399.0eV [see Fig. 11(a)] that can be fit to a broad pseudo-Voight function with a FWHM $=1.4 \mathrm{eV}$. The $\mathrm{N} 1 \mathrm{~s}$ binding energy (BE) and the broad width of the peak are consistent with a range of calculated sites that are likely due to two- and three-coordinated nitrogen in substitutional carbon sites that are bonded to interfacial silicon and carbon atoms [see supplemental material]! ${ }^{21}$ In these studies the integrated N1s XPS intensity corresponds to starting nitrogen concentration of $\sim 0.3 \mathrm{ML}\left(\sim 3.8 \times 10^{14} \mathrm{~cm}^{-2}\right.$ of $\mathrm{N}$ on the $4 \mathrm{H}-\mathrm{SiC}(0001)$ surface). As the XPS data of Fig. 1 will show, a large fraction of this $\mathrm{N}$ remains at the $\mathrm{SiC}$-graphene interface after graphene growth at $1450{ }^{\circ} \mathrm{C}$ in the controlled silicon sublimation (CSS) furnace [see supplemental material]. ${ }^{23}$ We suggest a model where a portion of the remaining nitrogen is not incorporated into the graphene but instead pins the graphene to the $\mathrm{SiC}$ to produce buckled semiconducting graphene with a finite bandgap.

After graphene growth, the graphene $\mathrm{C}$ 1s peak develops at $284.5 \mathrm{eV}$ [see Fig. 11(b)]. We note that both the Si 2p and $\mathrm{SiC} \mathrm{C1s} \mathrm{peaks} \mathrm{shift} 1.3 \mathrm{eV}$ to lower binding energy once the graphene forms [see Figs. 1(a) and (c)]. This $1.3 \mathrm{eV}$ shift 

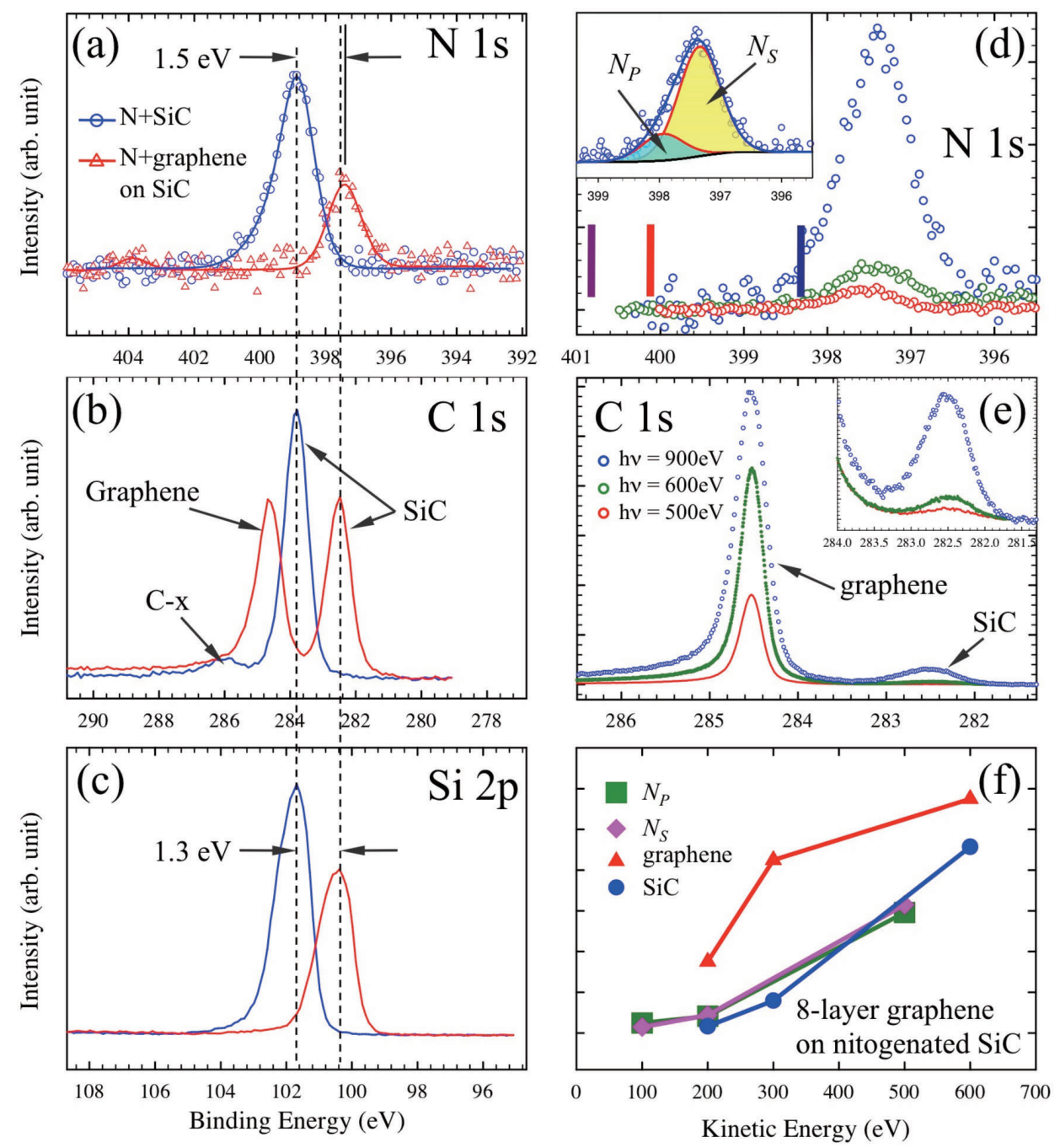

FIG. 1. (a)-(c) XPS from $\mathrm{SiC}(000 \overline{1})$ surface before and after graphene growth of a 3-layer graphene film. The pre- and post-growth nitrogen coverage is $0.3 \mathrm{ML}$ and $0.2 \mathrm{ML}$, respectively (a) N1s, (b) $\mathrm{C} 1 \mathrm{~s}$, and (c) $\mathrm{Si} 2 \mathrm{p}[h v=1486 \mathrm{eV}]$. A $1.3 \mathrm{eV}$ shift after graphene forms is shown in all three spectra. The C-x peak at $286 \mathrm{eV}$ is from surface "adventitious carbon" contamination in the initial nitrogen-seeded surface ${ }^{22}$ (d) and (e) XPS spectra for an 8-layer graphene film grown from the pre-nitrogen $\mathrm{SiC}$ surface using photon energies of $h v=500,600,900 \mathrm{eV}$ photons. (d) The N1s spectrum at the same photon energies as (e). Insert shows the 2-component fit to the N 1s peak. The purple, red and blue bars in (d) mark the expected peak positions of pyrrolic, graphitic and pyridinic N-inclusions in graphene, respectively [see Fig. 2] [] 7117 (e) XPS of the C1s spectrum for the same photon energies in (d). The insert in (e) shows the SiC portion of the C1s XPS spectra. (f) A comparison of the trend in photoelectron intensity versus photoelectron kinetic energy from an 8-layer graphene sample. The SiC C1s, graphene C1s, and the two N 1s XPS peaks are plotted. Absolute intensities have been scaled by arbitrary factors to highlight the energy trend for each peak.

is consistent with the known band bending on graphitized Cface $\mathrm{SiC} .[24[25]$ After growth, the N1s peak is shifted by $1.5 \mathrm{eV}$ to lower binding energy (a net shift of $-0.2 \mathrm{eV}$ ) and the total nitrogen concentration, as determined by XPS, reduces to $\sim$ $2.1 \times 10^{14} / \mathrm{cm}^{2}, \sim 0.2 \mathrm{ML}$ [see Experimental Methods]. The post growth $\mathrm{N}$ 1s peak can be fit with two narrow pseudoVoight peaks at $398.0 \mathrm{eV}$ and $397.4 \mathrm{eV}$ (with FWHM of 0.8 and $0.9 \mathrm{eV}$ ), labeled as $N_{P}$ and $N_{S}$, respectively [see the insert in Fig. 1(d)]. It is important to note that there are no other higher $\mathrm{BE} \mathrm{N}$ 1s peaks in the spectrum that would normally be associated with common nitrogen incorporation sites in the graphene lattice [see Figs. 2 and 1(d)] ${ }^{7]}$ In other words, little if any nitrogen is incorporated into the graphene lattice.
The dominant $\mathrm{N}$ 1s peak at $397.4 \mathrm{eV}$ is usually associated with $\mathrm{N}-\mathrm{SiC}$ bonds. Specifically, nitrogen in carbon sites bonded to silicon atoms at the $\mathrm{SiC}$ interface as shown in Fig. 3.a) [see supplemental material]. ${ }^{26}$ The weak $\mathrm{N}_{P}$ peak at $398.9 \mathrm{eV}(\sim 20 \%$ of the total nitrogen coverage) is in the energy range associated with pyridinic nitrogen sites in graphene [see Fig. 2[a)] ${ }^{[56 / 21 / 27]}$ but can also be associated with other $\mathrm{sp}^{3}$ and $\mathrm{sp}^{2} \mathrm{C}-\mathrm{N}$ bonds in carbon nitride films. ${ }^{28}$ However, photon energy-dependent XPS measurements in Figs. 11.d),(e), and (f) and STM images described below indicate that the $\mathrm{N}_{P}$ is not pyridinic nitrogen but is instead a second $\mathrm{C}-\mathrm{N}$ compound at the graphene-SiC interface that pins the graphene to the $\mathrm{SiC}$ interface [see Fig. 3(a)]. 


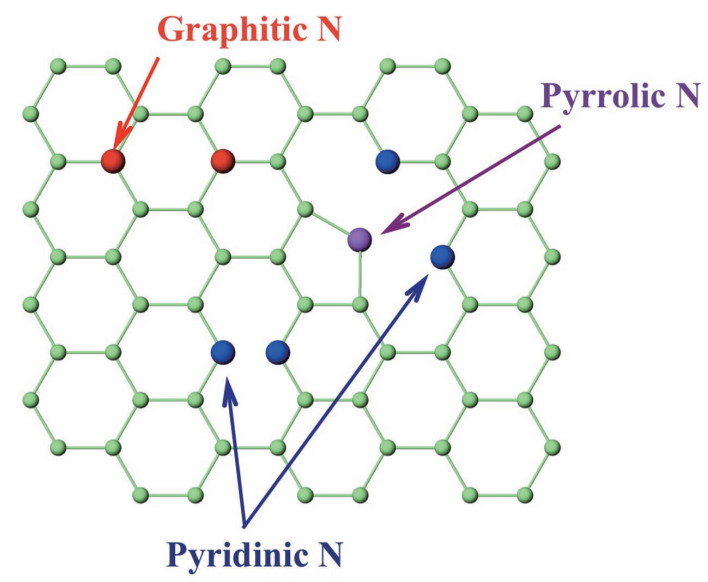

FIG. 2. (a) The three common nitrogen sites: quaternary (or graphitic), pyridinic, and pyrrolic.

Figures 1.d) and (e) show XPS spectra of N1s and C1s for different photon energies. A photon energy of $h v=500 \mathrm{eV}$ produces photoelectrons with the shortest mean free path and is therefore the most surface sensitive. This is evident by the weak $\mathrm{SiC} \mathrm{C} \mathrm{1s} \mathrm{peak} \mathrm{in} \mathrm{Fig.} \mathrm{1(e)} \mathrm{that} \mathrm{is} \mathrm{attenuated} \mathrm{by} \mathrm{the}$ graphene above the surface. The N1s peak is similarly the weakest at this energy indicating that most of the nitrogen is near the graphene-SiC interface. We also note that the ratio of the $N_{S}$ and $N_{P}$ peak intensities is independent of photon energy. This is demonstrated in Fig. 1.f) where the intensity of the two peaks have essentially the same kinetic energy dependence. Furthermore, a comparison of the $N_{S}$ and $N_{P}$ peaks measured at $h v=500 \mathrm{eV}$ for 3- and 8-layer graphene films, shows that the relative XPS intensities for the two thicknesses can be completely accounted for by the photoelectron attenuation through the different number of graphene layers. These two findings confirm that the $N_{P}$ peak is associated with a nitrogen site at the $\mathrm{SiC}$-graphene interface and is not a pyridinic nitrogen inclusion in graphene.

The XPS results shown in Fig. 11 (a) make clear that while a significant portion of the nitrogen desorbs during the high temperature $\left(1450{ }^{\circ} \mathrm{C}\right)$ growth, the remaining nitrogen is maintained in a stable concentration of sites at the grapheneSiC interface. This implies that any high energy substitutional nitrogen sites that develop in the growing graphene film are annealed out and remain bonded at more favorable sites at the $\mathrm{SiC}$ interface. Assuming a uniform distribution of both the $N_{S}$ and $N_{P}$ sites, the average distance between nitrogen atoms is $\sim 0.7 \mathrm{~nm}$ for $N_{S}$ sites and $\sim 1.4 \mathrm{~nm}$ for $N_{P}$ sites. A length scale comparable to the $N_{P}$ average spacing will be seen again in STM results from these same films and will be important in understanding the measured band structure of this material.

The interfacial nitrogen preparation method used here causes a band-gap to open in the graphene $\pi$ bands. We demonstrate this using high resolution ARPES measurements of the graphene films taken at the graphene $K$-point, which is rotated $30^{\circ}$ from the $\mathrm{SiC} K$-point. Figure 4 (a) shows the typical band dispersion perpendicular to the $\Gamma K$ direction at the graphene $K$-point from a clean 3 -layer graphene film. As the ARPES shows, pristine 3-layer C-face graphene consists of the linear $\pi$-bands (Dirac cones) with little or no doping. 29 The graphene band structure of a 3-layer film grown from a $0.2 \mathrm{ML}$ interface nitrogen content is dramatically different as seen in Fig. 4(b) and (c). When interfacial nitrogen is present, a band-gap develops. Figure 4(d) shows energy distribution curves (EDC) through the $K$-point at $k_{y}=0$ from (a), (b), and (c). The EDC for the clean surface is peaked near the $E_{F}$ (the small intensity at $0.5 \mathrm{eV}$ is from a faint cone from the layer below rotated relative to the top layer, typical of $\mathrm{C}$ face graphene) ${ }^{30}$ Unlike the pristine graphene, the peak in the EDCs from the nitrogen-seeded samples are shifted to higher binding energies indicating a valence band maximum corresponding to an energy gap. Figure 4(d) shows that the 3-layer nitrogen-seeded sample has a gap energy of at least $0.7 \mathrm{eV}$ (depending on the position of the conduction band minimum), while the 8-layer graphene film has a smaller gap of $0.45 \mathrm{eV}$ (a 4-layer film, not shown, confirms this trend). Carbon 1s core level spectra from clean and from all the nitrogen-seeded samples show that the valence band spectra cannot be due to a simple shift of the graphene Dirac cone to higher BE caused by band bending changes in the different samples. We also note that the effective Fermi velocity $\left(\tilde{v}_{F}\right)$ is reduced, consistent with the opening of a band-gap. Both the 3-and 8-layer samples have $\tilde{v}_{F}=0.8 \pm 0.05 \times 10^{6} \mathrm{~m} / \mathrm{sec}$.

For both 3- and 8-layer films, the valence band maximum edge and the $\Delta k$-width of the $\pi$ bands are broader in the nitrogen-seeded sample compared to pristine graphene. The momentum broadening is seen more clearly in momentum distribution curves (MDC) taken at $E-E_{F}=-1.5 \mathrm{eV}$ [ see Fig. 4(e)]. A fit to the MDC with two Lorentzians gives a FWHM $\Delta k \sim 0.25 \AA^{-1}$ (independent of layer thickness). A large part of the energy broadening in the EDCs and momentum broadening in the MDCs is due to the corrugation of the graphene surface [the corrugation is demonstrated in STM results presented below]. Small modulations in the local graphene height cause a local angular variation in the surface normal. Since the surface normal determines the orientation of the graphene Brillouin zone, the corrugated surface leads to local $k_{x}$ and $k_{y}$ shifts in the $K$-point. This leads to an ARPES image that is an area average of a distribution of parabolic cuts through Dirac cones from local tilted graphene that results in an $E$ - and $k$-broadened ARPES spectrum. The corrugations in the graphene explain part of the broad intensity distribution between the valence band maximum and $E_{F}$. A more detailed STM analysis suggest how the gap forms and why the EDC distribution is broad.

STM images of the nitrogenated graphene show that the films are not flat like typical C-face graphene films. ${ }^{[1]}$ Figures 5(a) and (b) show the highly buckled topography. A close up view of the buckled regions in Fig. 5. (c) show that it is made up of folded, intact graphene. Note that there are no indications of nitrogen inclusions like those seen in CVD and plasma created nitrogen $17 / 32$ The folds are $\sim 1 \mathrm{~nm}$ (no more than $2 \mathrm{~nm}$ ) high and $2-4 \mathrm{~nm}$ wide giving a radius of curvature of $1-2 \mathrm{~nm}$. These folds meander and can extend up to $25 \mathrm{~nm}$ but are typically $5 \mathrm{~nm}$ long. At higher magnification one sees the honeycomb structure over a fold indicating they are part of 


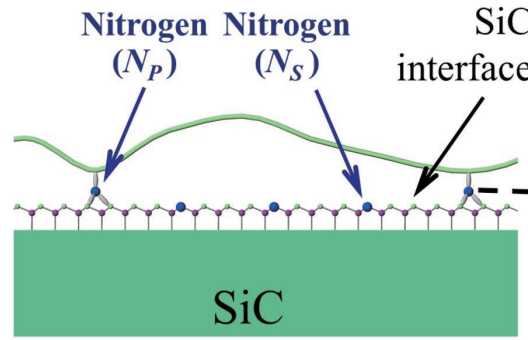

(a)

$\mathrm{SiC}$

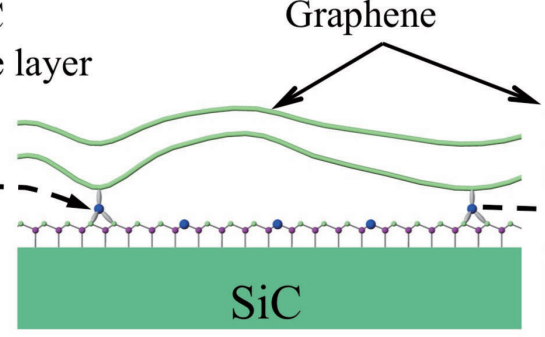

(b)

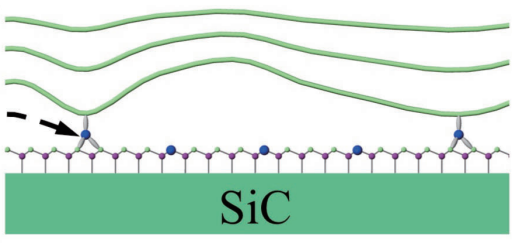

(c)

FIG. 3. (a), (b) and (c) Schematics showing how graphene layers grow from nitrogen-seeded SiC. (a) shows that most of the nitrogen is bonded to $\mathrm{Si}$ atoms (magenta) in carbons sites, $N_{S}$, sites. The remaining interfacial nitrogen, $N_{P}$, is bonded to both the $\mathrm{SiC}$ carbon atoms (green) and the graphene, pinning it to the $\mathrm{SiC}$. As the $\mathrm{SiC}$ recedes and the graphene grows, nitrogen remains at the $\mathrm{SiC}$ interface. (b) and (c) both show that the buckling amplitude in layers farther from the surface is reduced as strain is relieved.
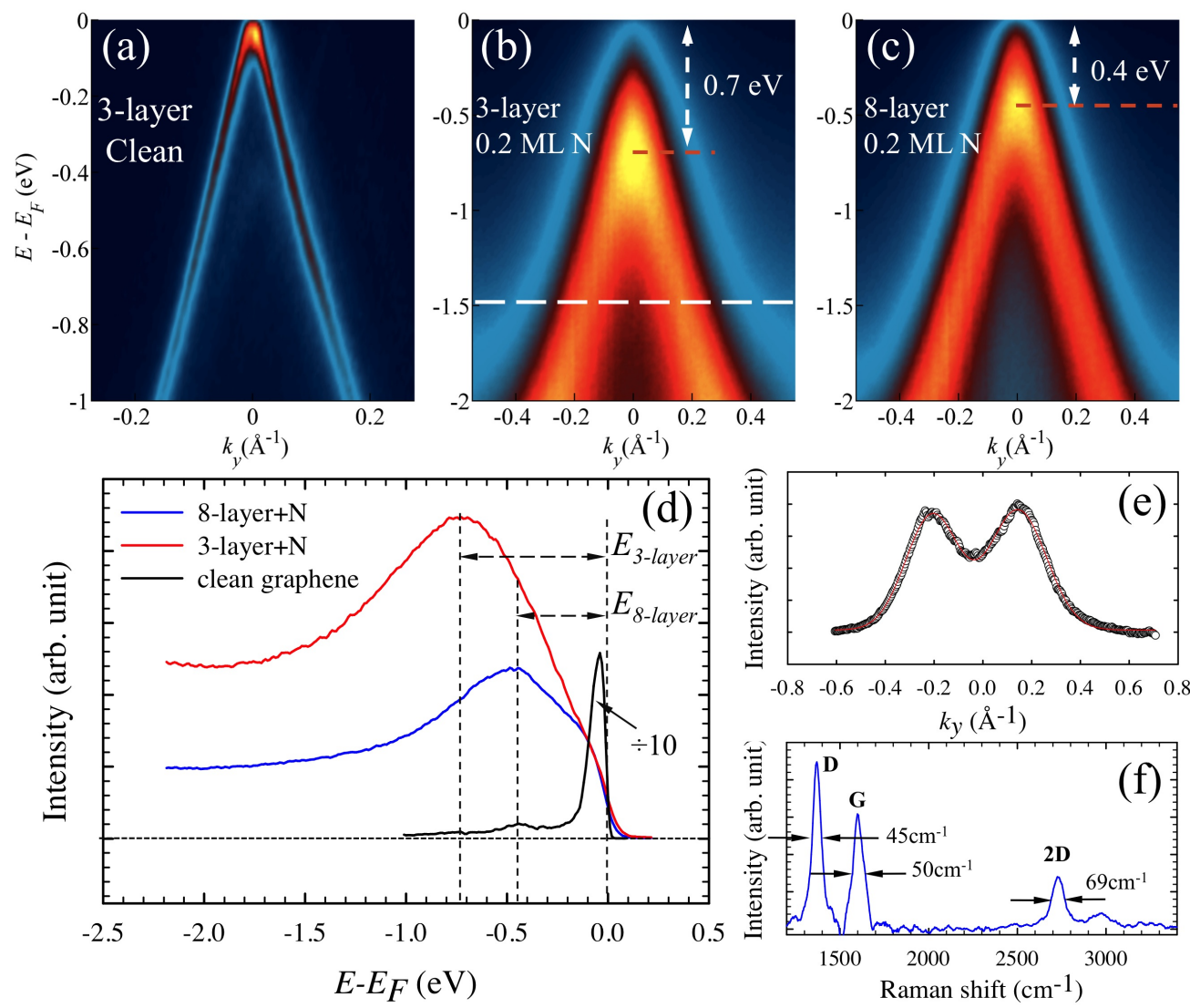

FIG. 4. (a), (b) and (c) are the ARPES band structure near the $K$-point of (a) clean 3-layer graphene (T=100K), (b) 3-layer graphene grown with a $0.2 \mathrm{ML}$ nitrogen $\mathrm{SiC}(000 \overline{1})$ surface $(\mathrm{T}=300 \mathrm{~K})$, and (c) 8-layer graphene grown from a $0.2 \mathrm{ML}$ nitrogen $\mathrm{SiC}(000 \overline{1})$ surface $(\mathrm{T}=300 \mathrm{~K})$. $k_{y}$ is perpendicular to the $\Gamma K$ direction. (d) Energy distribution curves (EDC) through the Dirac point in (a), (b) and (c) show the $0.7 \mathrm{eV}$ gap for 3-layer films that reduces to $0.4 \mathrm{eV}$ for 8-layer films. (e) MDC made at $\mathrm{BE}=-1.5 \mathrm{eV}$ from the Dirac cone in (b) (circles). A double Gaussian peak fit (red line) is shown. (f) Raman spectra from a nitrogen grown 3-layer graphene film after subtracting the contribution from the SiC. $\mathrm{N}$ concentration is $0.2 \mathrm{ML}$.

a continuous graphene sheet and not isolated graphene grains. Figure 5(d) shows an STM topographic image of a region with several folds. A $d I / d V$ image of a small region between folds in Fig. 5(e) shows a continuous density of states pattern confirming that the graphene is continuous.

It is important to point out that the $2-4 \mathrm{~nm}$ width of these folds is consistent with the $0.25 \AA^{-1} \Delta k$ broadening of the ARPES spectrum in Figs. 4(b) and (c). Since $\Delta k$ in ARPES is inversely proportional to the average coherent domain size ( $\Delta k=2 \pi / L)$, we can estimate the ordered graphene regions to be $\sim 2.5 \mathrm{~nm}$. In addition to the scale of the finite size domains measured by ARPES, the mean separation between $N_{P}$ 


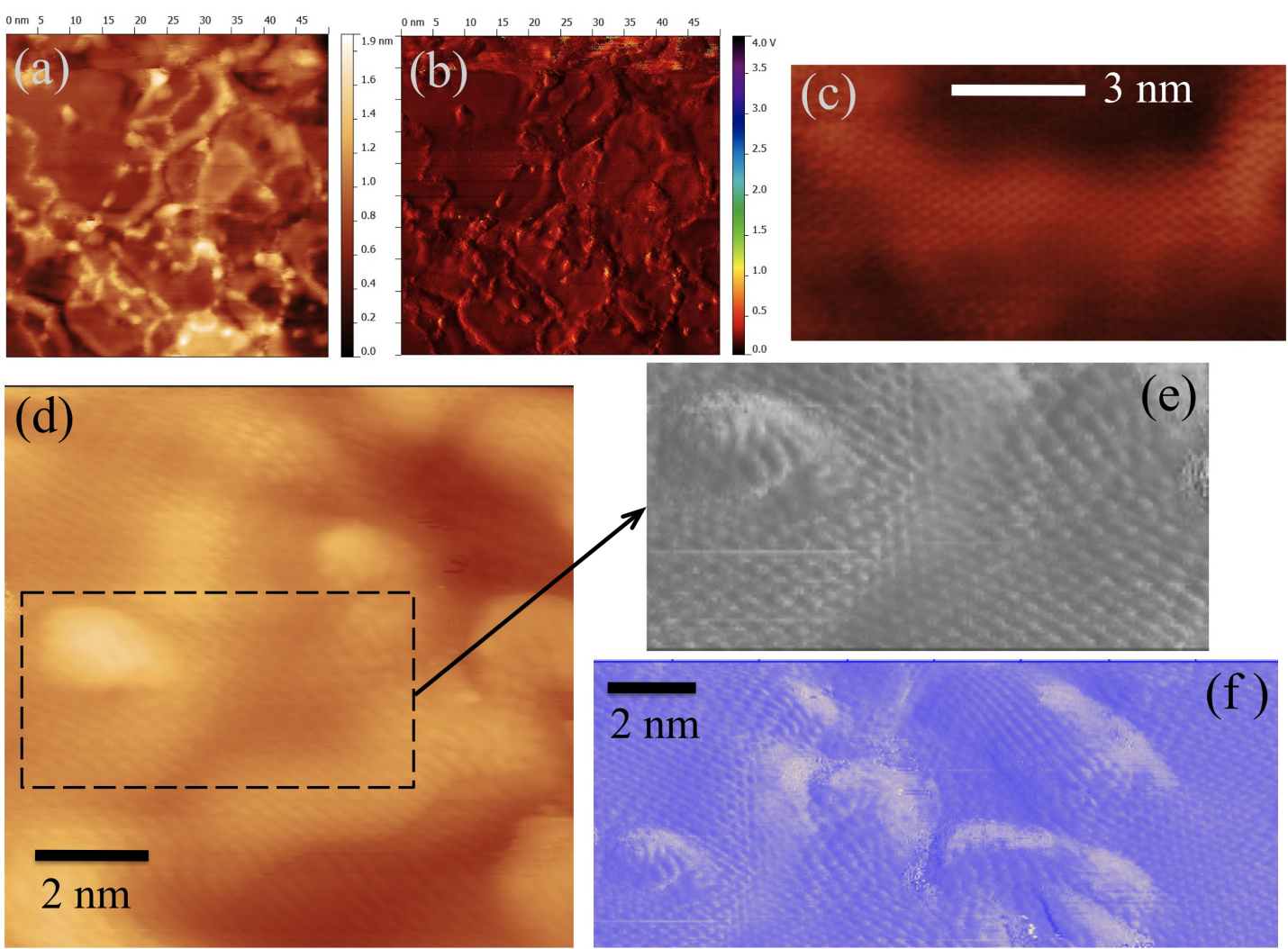

FIG. 5. (a) $50 \times 50 \mathrm{~nm}^{2} \mathrm{STM}$ image of a 3-layer graphene film grown from a $0.2 \mathrm{ML}$ nitrogen-seeded $\mathrm{SiC}(000 \overline{1})$ surface (bias voltage, $V_{B}=0.1 \mathrm{~V}$ ). The image shows a pattern of folds. (b) A $d I / d V$ image of (a) $\left(V_{B}=0.1 \mathrm{~V}\right.$ ). (c) A topographic image of a fold showing the graphene lattice $\left(V_{B}=0.1 \mathrm{~V}\right)$. (d) A $10 \times 10 \mathrm{~nm}$ view of the graphene folds. (e) and (f) are magnified $d I / d V$ images of folded graphene in (d). The image in (e) shows that the graphene is continuous over the folds and in the valleys. (f) shows that the tops of the folds are bright in the $d I / d V$ image indicating a high density of states expected in strained graphene.

nitrogen atoms, as determined by XPS, is $1.4 \mathrm{~nm}$; only slightly smaller than the width of the folds determined by STM. Raman spectra from the graphene show broad G- and 2D-peaks typical of multilayer C-face graphene [see Fig. 4ff)]. However, the large $\mathrm{D}$-peak is an indication of significant $\mathrm{sp}^{3}$ bonds, consistent with the $N_{P}$ XPS peak associated with graphene-N bonds. However, quantitative Raman does not allow us to estimate the number of such bonds. In fact a typical estimate of the graphene domain size, using the Raman G- and D- peak ratio, over estimates the actual fold size by an order of magnitude [see supplement].

That fact that three different techniques measure similar length scales is not a coincidence. A model consistent with these results is shown in Fig. 3. During the high temperature growth, Si evaporates leaving a carbon rich surface with nitrogen impurities. As the graphene layer crystalizes from this film at high temperature, nitrogen atoms in high energy interstitial graphene sites are expelled from the growing graphene layer. Some of these nitrogen desorb while the others re-bind to the $\mathrm{SiC}$ as either $N_{S}$ or $N_{P}$ nitrogen [see Fig. 3 a)]. The interfacial nitrogen atoms $\left(N_{P}\right)$ act as pinning sites that lock the graphene to the SiC. These $\mathrm{sp}^{3}$ bonded C-N-SiC sites constrain the graphene to a length scale related to the starting ni- trogen concentration. Since the nitrogen constrained graphene is no longer commensurate with the $\mathrm{SiC}$, a strain develops in the film that forces the graphene to buckle. These folds are similar to the differential thermal expansion induced strain pleats normally observed in epitaxial graphene, 33 although the folds in this study are much smaller in both length and width. Unlike the pleats, the length scale between folds is now set by the strain field induced by the interfacial nitrogen concentration. As the next graphene layer forms, Si evaporation causes the $\mathrm{SiC}$ interface to recede along with the interfacial nitrogen as shown in Figs. 3(b) and (c). This is again consistent with the XPS results that show the same post growth interfacial nitrogen concentration whether or not 4- or 8-layers of graphene are grown from the same starting nitrogen concentration. As each new graphene layer forms the process is repeated. This leaves a stack of $\pi$-bonded graphene layers with only the last layer nitrogen bonded to the $\mathrm{SiC}$. The buckling period remains determined by the nitrogen concentration and not the number of layers (confirmed by the layer independent ARPES broadening). As Fig. 3 shows, the buckling amplitude is expected to reduce in layers farther from the $\mathrm{SiC}$ as previously shown by STM! 35

It is these folds that are responsible for the energy gap 
and not nitrogen impurities in the graphene. Because of the short electron mean free path, the ARPES only measures the graphene band structure in the top 2-3 layers where the XPS shows no measurable nitrogen concentration. This means that the ARPES measured band-gap must be due to the folds in the graphene. Specifically, the size of the ARPES measured gap and its dependence on the layer thickness point to a finite size effect gap caused by either strain confined domain boundaries or by a quasi periodic strain field.

The boundary between folds is a highly strained region of graphene that could confine the graphene wave functions to the flat portion of the fold. The confinement band-gap expected for a graphene ribbon with width $w=1-2 \mathrm{~nm}$ is $E_{g} \sim 1 \mathrm{eV}-\mathrm{nm} / w=1.0-0.5 \mathrm{eV}, \underline{20 \mid 36}$ which is close to the value measured. The fact that a distribution of confined folds are part of a continuous sheet of graphene would also explain the ARPES intensity seen within the gap. A model of bent graphene confined by flat graphene sheets predicts a significant density gap due to boundary resonant states. ${ }^{12}$ The finite size model also explains the smaller gap in thicker graphene films even though the domain size is the same. As the number of layers increases the amplitude of the buckles is reduced so that the strain at the bends is also reduced. This makes the confinement boundary less well defined and effectively increases the confined region, thus reducing the band-gap. Also, because there is a distribution of fold widths in the film, we would expect that the area averaged ARPES would have a distribution of band-gaps. This would account for the intensity between the top of the valance band and $E_{F}$ seen in Fig. 4(d).

Periodic strain fields are also expected to open a bandgap 19 The folds are strained and show the typical increased density of states expected for strained folds. Figure 5 (e) shows a $d I / d V$ image of several fold ridges. The ridge becomes bright at their apex indicating a high density of states that is predicted in strained graphene ${ }^{34}$ and observed in STM on graphene nano-bubbles. 37 While the nitrogen induced folds are not strictly periodic, the folds have an average separation of about 2-4 $\mathrm{nm}$. This is a very small period that has the potential to open a large gap. Whether strain or electron confinement (or both) are responsible for the observed gap remains to be determined.

We have shown that a partial nitrogen monolayer, pregrown on the $\mathrm{SiC}(000 \overline{1})$ surface, can be used to form an undulating graphene layer with a band-gap $\sim 0.7 \mathrm{eV}$. The nitrogen binds the growing graphene to the $\mathrm{SiC}$ interface forming $1-2 \mathrm{~nm}$ high ridges in the graphene. There is no nitrogen intercalation between graphene sheets nor is there evidence of nitrogen inclusions that can reduce graphene's mobility 3839 Because the initial nitrogen-carbon bond is stable to very high temperatures, the pre-grown layer can be patterned to produce locally strained graphene. This semiconducting form of graphene would then be seamlessly connected to metallic graphene grown from the non-nitrogenated $\mathrm{SiC}$. It offers a potential way to produce all graphene semiconductor-metal junctions.

\section{ACKNOWLEDGMENTS}

This research was supported by the NSF under Grants No. DMR-1206793, DMR-1206655, and DMR-1206256, Additional support is also acknowledged from the NSF DMR1005880 and the W.M. Keck Foundation. We thank A. Savu for growing the graphene films and Z. Dromsky for taking the Raman spectra. We also wish to acknowledge the SOLEIL synchrotron radiation facilities and the Cassiopée beam line staff. We wish to thank N. Guisinger for his assistance in the STM measurements. STM and STS experiments were done at the Center for Nanoscale Materials supported by the U. S. Department of Energy, Office of Science, Office of Basic Energy Sciences, under Contract No. DE-AC02-06CH11357. We thank Profs. J. Williams and S. Dher for useful discussions concerning the NO proceess.

\section{EXPERIMENTAL METHOD}

The substrates used in these studies were n-doped (nitrogen) $4 \mathrm{H}-\mathrm{SiC}$. All the graphitized samples were grown in a closed RF induction furnace using the Confined Silicon Sublimation (CSS) method as described below! 23 The samples were transported in air before introduction into either the XPS, STM, or the ARPES UHV chamber. ARPES and photon energy dependent XPS measurements were made at the Cassiopée beamline at the SOLEIL synchrotron in Gif sur Yvette. The high resolution Cassiopée beamline has a resolution $E / \Delta E \simeq 70000$ at $100 \mathrm{eV}$ and 25000 for lower energies. The detector is $\mathrm{a} \pm 15^{\circ}$ acceptance Scienta $\mathrm{R} 4000$ detector with resolution $\Delta E<1 \mathrm{meV}$ and $\Delta k \sim 0.01 \AA^{-1}$ at $\hbar \omega=36$ $\mathrm{eV}$. The total measured instrument resolution is $\Delta E<12 \mathrm{meV}$.

To produce the initial nitrogen surface layers the $\mathrm{SiC}$ substrates were RCA cleaned. The samples were loaded into a $900{ }^{\circ} \mathrm{C}$ furnace under a $500 \mathrm{sccm} \mathrm{Ar}$ flow and heated to $1175{ }^{\circ} \mathrm{C}$ over a $1 \mathrm{hr}$ ramp. The sample is then kept at $1175{ }^{\circ} \mathrm{C}$ for $2 \mathrm{hrs}$ (for a $0.3 \mathrm{ML}$ nitrogen coverage) with a $500 \mathrm{sccm}$ NO flow ${ }^{40}$ The sample is then cooled to $900{ }^{\circ} \mathrm{C}$ under a $500 \mathrm{sccm}$ Ar flow and unloaded from furnace. Oxide grown through this anneal is removed by HF immediately before graphene growth. These C-face nitrogen-seeded surfaces were then heated in a closed graphite crucible in an $\mathrm{RF}$ vacuum furnace to $1450{ }^{\circ} \mathrm{C}$ to produce the graphene films. This growth temperature is slightly higher that the desorption temperature of nitrogen as discussed in the supplement.

The nitrogen coverage, $N_{N}$, is estimated from the ratio of the $\mathrm{N}$ 1s to Si 2p intensities $N_{N}=\frac{I_{N}}{I_{S i}} \frac{\sigma_{S i}}{\sigma_{N}} n_{S i} \lambda$, where $I_{N}$ and $I_{S i}$ are the $\mathrm{N} 1 \mathrm{~s}$ and $\mathrm{Si} 2 \mathrm{p}$ XPS intensities, $\sigma_{N}$ and $\sigma_{S i}$ are the photoionization cross sections of $\mathrm{N}$ and $\mathrm{Si} .^{4142} n_{S i}=4.8 \times$ $10^{22} / \mathrm{cm}^{3}$ is atomic density of $\mathrm{Si}$ in $\mathrm{SiC}$. For this work, we use a mean free path in $\mathrm{SiC}$ of $\lambda=2.2 \mathrm{~nm}$ at $1486 \mathrm{eV}$. 
${ }^{1}$ L. Kubler, K. Aït-Mansour, M. Diani, D. Dentel, J.-L. Bischoff, and M. Derivaz, Phys. Rev. B 72, 115319 (2005).

2 I. Gierz, C. Riedl, U. Starke, C.R. Ast, and Klaus Kern, Nano Lett. 8, 4603 (2008).

3 C. Coletti, C. Riedl, D-S. Lee, B. Krauss, K. von Klitzing, J. Smet and U. Starke, Phys. Rev. B 81, 235401 (2010).

4 K.V. Emtsev, A.A. Zakharov, C. Coletti, S. Forti, U. Starke, Phys. Rev. B 84, 125423 (2011).

5 D. Usachov, O. Vilkov, A. Gr'uneis, D. Haberer, A. Fedorov, V. K. Adamchuk, A. B. Preobrajenski, P. Dudin, A. Barinov, M. Oehzelt, C. Laubschat, and D. V. Vyalikh, Nano Lett. 11, 5401 (2011).

6 E. Velez-Fort, C. Mathieu, E. Pallecchi, M. Pigneur, M. G. Silly, R. Belkhou, M. Marangolo, A. Shukla, F. Sirotti, and A. Ouerghi, ACS Nano 6, 10893 (2012).

7 H. Wang, T. Maiyalagan, and X. Wang, ACS Catal. 2, 781 (2012).

8 M.Y. Han, J.C. Brant, and P. Kim, Phys. Rev. Lett. 104, 056801 $1-4(2010)$.

9 L. Jiao, L. Zhang, X. Wang, G. Diankov, and H. Dai, Nature 458, 877-880 (2009).

${ }^{10}$ L. Jiao, X. Wang, G. Diankov, H. Wang, and H. Dai, Nature Nanotech. 5, 321-325 (2010).

11 J. B. Oostinga, B. Sacépè, M. F. Craciun, and A. F. Morpurgo, Phys. Rev. B 81, 193408 1-4 (2010).

12 J. Hicks, A. Tejeda, A. Taleb-Ibrahimi, M.S. Nevius, F. Wang, K. Shepperd, J. Palmer, F. Bertran, P. Le Fèvre, J. Kunc, W.A. de Heer, C. Berger, and E.H. Conrad, it Nature Phys. 9, 49 (2013).

13 J. Baringhaus, C. Tegenkamp, F. Edler, M. Ruan, E. Conrad, C. Berger, W.A. de Heer, arXiv:1301.5354 (2013).

14 S. Niyogi, E. Bekyarova, M.E. Itkis, H. Zhang, K. Shepperd, J. Hicks, M. Sprinkle, C. Berger, C. N. Lau, W.A. deHeer, E.H. Conrad, and R.C. Haddon, Nano Lett. 10, 4061 (2010).

15 R. Balog, B. Jørgensen, L. Nilsson, M. Andersen, E. Rienks, M. Bianchi, M. Fanetti, E. Lægsgaard, A. Baraldi, S. Lizzit, Z. Sljivancanin, F. Besenbacher, B. Hammer, T.G. Pedersen, P. Hofmann and L. Hornekær, Nature Mat. 9, 315 (2010).

16 M. Deifallah, P.F. McMillan, F. Cora, J. Phys. Chem. C 112, 5447 (2008).

17 F. Joucken, Y. Tison, J. Lagoute, J. Dumont, D. Cabosart, B. Zheng, V. Repain, C. Chacon, Y. Girard, A. R. Botello-Méndez, S. Rousset, R. Sporken, J-C. Charlier, and L. Henrard, Phys. Rev. B 85, 161408(R) (2012).

18 A. Lherbier, X. Blase, Y.M. Niquet, F. Triozon, S. Roche, Phys. Rev. Lett. 101, 036808 (2008).

19 T. Low, F. Guinea, and M. I. Katsnelson, Phys. Rev. B 83, 195436 (2011).

20 K. Wakabayashi, K.-ichi Sasaki, T. Nakanishi, and T. Enoki, Sci. Technol. Adv. Mater. 11, 054504-054521 (2010).

21 A. Snis and S.F.Matar, Phys.Rev. B 60,10855 (1999).

22 A. Avila, I. Montero, L. Galán, J. M. Ripalda, and R. Levy, J. Appl. Phys. 89, 212 (2001).

23 W.A. de Heer, C. Berger, M. Ruan, M. Sprinkle, X. Li, Y. Hu, B. Zhang, J. Hankinson, and E.H. Conrad, Proc. Nat. Acad. Sci. 108, 16900 (2011).

24 T. Seyller, K. Emtsev, F. Speck, K.-Y. Gao, and L. Ley, Mater. Sci. Forum 556-557, 701 (2007).

25 K.V. Emtsev, F. Speck, Th. Seyller, L. Ley, and J. D. Riley, Phys. Rev. B 77, 155303 (2008).

26 H. Tochihara and T. Shirasawa, Prog. in Surf. Sci. bf 86, 295 (2011).

27 I. Shimoyama, G. Wu, T. Sekiguchi, and Y. Baba, Phys. Rev. B
62, R6053 (2000).

28 C. Ronning, H. Feldermann, R. Merk, H. Hofsass, P. Reinke, and J.U. Thiele, Phys. Rev. B 58, 2207 (1998).

29 J. Hicks, K. Shepperd, F. Wang and E. H. Conrad, J. Phys. D: Appl. Phys. 45, 154002 (2012).

30 M. Sprinkle, D. Siegel, Y. Hu, J. Hicks, A. Tejeda, A. TalebIbrahimi, P. Le Fèvre, F. Bertran, S. Vizzini, H. Enriquez, S. Chiang, P. Soukiassian, C. Berger, W.A. de Heer, A. Lanzara, E.H. Conrad, Phys. Rev. Lett. 103, 226803 (2009).

31 D.L. Miller, K.D. Kubista, G.M. Rutter, M. Ruan, W.A. de Heer, M. Kindermann, P.N. First, and J.A. Stroscio, Nature Phys. 6, 811 (2010).

32 L. Zhao, R. He, K.T. Rim, T. Schiros, K.S. Kim, H. Zhou, C. Gutièrez, S.P. Chockalingam, C.J. Arguello, L. Páová D. Nordlund, M.S. Hybertsen, D.R. Reichman, T.F. Heinz, P. Kim, A. Pinczuk, G.W. Flynn, and A.N. Pasupathy, Science 333, 999 (2011).

33 J. Hass, W.A. de Heer and E.H. Conrad, J. Phys.: Condens. Matt. 20, 323202 (2008)

34 V.M. Pereira, A. H. Castro Neto, H.Y. Liang, and L. Mahadevan, Phys. Rev. Lett. 105, 156603 (2010).

35 C. Riedl, U. Starke, J. Bernhardt, M. Franke, and K. Heinz, Phys. Rev. B 76, 245406 (2007).

36 Y.-W. Son, M.L. Cohen, and S.G. Louie, Phys. Rev. Lett. 97, $2168031-5$ (2006).

37 N. Levy, S. A. Burke, K. L. Meaker, M. Panlasigui, A. Zettl, F. Guinea, A. H. Castro Neto, and M. F. Crommie, Science 329, 544 (2010).

38 D. Wei, Y. Liu, Y. Wang, H. Zhang, L. Huang, and G. Yu, Nano Lett. 9, 1752 (2009).

39 Z. Jin, J. Yao, C. Kittrell, and J.M. Tour, ACS Nano 5, 4112 (2011).

40 G. Liu, A.C. Ahyi, Y. Xu, T. Isaacs-Smith, Y.K. Sharma, J.R. Williams, L.C. Felman, and S. Dhar, IEEE Elec. Device Lett. 34, $181(2013)$

41 J.-J. Yeh and I. Lindau, Atomic Data Nucl. Data Tables 32, 1 (1985).

42 J.-J. Yeh, Atomic Calculations of Photoionization Cross Sections and Asymmetry Parameters, (Gordon and Breach, Langhorne, PA, 1993). 